\title{
What is known about the influence of dentine hypersensitivity on oral health-related quality of life?
}

\author{
Katrin Bekes • Christian Hirsch
}

Received: 1 December 2011 / Accepted: 21 November 2012 /Published online: 6 December 2012

(C) Springer-Verlag Berlin Heidelberg 2012

\begin{abstract}
Objectives Oral health-related quality of life is a relatively new but rapidly growing concept in dentistry. It is an aspect of dental health addressing the patient's perception of whether his/her current oral health status has an impact upon his/her actual quality of life. Dentine hypersensitivity (DHS), which is a common condition of transient tooth pain associated with a variety of exogenous stimuli, may disturb the patient during eating, drinking, toothbrushing and sometimes even breathing. The resulting restrictions on everyday activities can have an important effect on the patient's quality of life. The aims of this paper were to consider the concept of oral health-related quality of life and to review and discuss the literature on oral healthrelated quality of life and DHS.

Material and methods A PubMed literature research was conducted using the terms ("dentin sensitivity" [MeSH Terms] OR ("dentin" [All Fields] AND "sensitivity" [All Fields]) OR "dentin sensitivity" [All Fields]) AND (("oral health" [MeSH Terms] OR ("oral" [All Fields] AND "health" [All Fields]) OR "oral health" [All Fields]) AND related [All Fields] AND ("quality of life" [MeSH Terms] OR ("quality" [All Fields] AND "life" [All Fields]) OR "quality of life" [All Fields])). Furthermore, a manual search was carried out. Any relevant work published presenting pertinent information about the described issue was considered for inclusion in the review.
\end{abstract}

K. Bekes $(\bowtie)$

Department of Operative Dentistry and Periodontology, Department of Pediatric Dentistry, Martin-Luther-University Halle-Wittenberg, University School of Dental Medicine, Harz 42a,

06108 Halle, Germany

e-mail: katrin.bekes@medizin.uni-halle.de

C. Hirsch

Department of Pediatric Dentistry, University of Leipzig, Liebigstraße $10-14$, House 1,

04103 Leipzig, Germany
Results The combination of the search terms resulted in a list of only three titles. The few published studies convincingly demonstrated that oral health-related quality of life is negatively affected in patients suffering from DHS.

Conclusions Patients with sensitive teeth report substantial oral health-related quality of life (OHRQoL) impairment. Nevertheless, knowledge about the influence of DHS on oral health-related quality of life is incomplete and, therefore, needs further research.

Clinical relevance Oral diseases can lead to physical, psychological and social disability. This paper shows that DHS can have a negative impact on the patients' OHRQoL.

Keywords Oral health-related quality of life (OHRQoL) . Dentine hypersensitivity · Oral Health Impact Profile (OHIP) . Dentine Hypersensitivity Experience Questionnaire (DHEQ)

\section{Introduction}

Quality of life, health-related quality of life and oral health-related quality of life

Quality of life (QoL) research has gained increasing attention in medicine and dentistry in recent years, undergoing a quantum shift. Where it was once regarded as a secondary outcome, occasionally useful to complement biologic and clinical markers of disease, QoL issues are now at the forefront of public health policy [1]. Quality of life can be defined as an individual's perception of his/her position in life, in the context of the culture and value systems in which he/she lives and in relation to his/her expectations, goals and concerns [2]. In 2003, Allen noted that "there appears to be an association between health and quality of life, which is not clearly defined, and the term health related quality of life is used to describe this association" [3]. Although the term health-related quality of life has no strict definition, there is 
consensus that it is a multidimensional construct capturing people's perceptions about factors that are important in their everyday lives [1].

Oral health-related quality of life (OHRQoL) is a part of health-related quality of life that focuses on oral health and orofacial concerns. It describes the way in which oral health affects a person's ability to function, psychological status, social factors and pain or discomfort [4]. Therefore, the OHRQoL attempts to represent the subjective side of oral health. Not surprisingly, as with health-related quality of life, the term OHRQoL has no strict definition [5]. However, it is generally agreed that it is also a multidimensional concept. The definitions for OHRQoL that are available vary from simple to more rigorous. One example of a simple definition is the one provided by the U.S. Surgeon General's report on oral health, which defines OHRQoL as "a multidimensional construct that reflects (among other things) people's comfort when eating, sleeping, and engaging in social interaction; their self-esteem; and their satisfaction with respect to their oral health" [6].

\section{Concepts of oral health}

Traditional methods to measure oral health are based on clinical standards. As in medicine, clinical dental indicators and indices of disease are used as an inverse measure of oral health in dentistry. These indices provide a quantitative method for measuring, scoring and analysing dental conditions in individuals and groups. An index describes the status of individuals or groups with respect to the condition to be measured. For example, the gingival index [7] and periodontal index [8] are used to describe periodontal diseases, and the DMFT index (number of decayed, missing and filled teeth) is used to describe a person's dental caries experience [9]. However, as important as these objective measures are, they only reflect the endpoint of the disease processes [3]. They also focus on the mouth rather than the person and give no indication of their impact on an individual's daily life and general health [5].

The limitations of this paradigm of oral health have been recognised, principally that this model only deals with disease [3]. Thus, Locker developed a conceptual framework for measuring oral health status that is based on the World Health Organization classification of impairment, disability and handicap and attempts to capture all possible functional and psychosocial outcomes of oral disorders [10]. In this model, disease can lead to impairment, which may then lead to a functional limitation or pain/discomfort, either physical or psychological. Either of these outcomes may lead to physical, psychological or social disability, which is defined by Locker as any limitation in or lack of ability to perform activities of daily living. As a final consequence, handicap can occur. It is characterised by social disadvantage, e.g. social isolation. Functional limitation may also lead directly to handicap [10].
Measuring oral health-related quality of life

OHRQoL can be measured using a questionnaire approach. Results can serve as an outcome measure; they allow insight into how a patient's oral health affects his/her well-being and quality of life at a given point in time [11]. Multipleitem questionnaires are the most widely used method to assess OHRQoL. Researchers have developed several quality of life instruments specific to oral health, and the number continues to grow rapidly to comply with the demand for more specific measures [5].

Slade and Spencer developed the Oral Health Impact Profile (OHIP), which differs from other measures in that it is based on both Locker's conceptual framework as well as input from dental patients with a variety of oral conditions [12]. The OHIP is the most widely used instrument in studies evaluating OHRQoL. The questionnaire attempts to measure the effects of both the frequency and the severity of oral problems on functional and psychosocial well-being. The OHIP has 49 items that are grouped into seven subscales: functional limitation, pain, psychological discomfort, physical disability, psychological disability, social disability and handicap. For each OHIP item, the patient is asked how frequently he/she experienced the impact of that item during last month. Responses concerning item impact are given using an ordinal rating scale $(0=$ never, $1=$ hardly ever, $2=$ occasionally, 3 =fairly often, $4=$ very often). The summary score (sum of item responses resulting in a range of $0-4 \times 49=0-196)$ represents a "problem index" that characterises the OHRQoL. A "O" summary score indicates the absence of any problems, higher OHIP scores represent more impaired OHRQoL, and a summary score of "196" indicates that all problems are experienced very often. The patient's score can be evaluated by comparison to a table of standard values representative of different populations.

The OHIP is a technically sophisticated OHRQoL instrument that is widely used internationally $[12,13]$. Several language versions already exist (e.g. Spanish [14], Swedish [15], Chinese [16], German [17] and Hungarian [18]) and initial evidence for the instrument's cross-cultural equivalence is available [14-18]. The questionnaire has been applied several times, including in patients with temporomandibular disorders [19, 20], xerostomia [21], burning mouth syndrome [22], tooth agenesis [23] and HIV infection [24, 25]. Clinical trials have also used the OHIP to evaluate implant-supported prostheses $[26,27]$ and steroidal therapy for oral lichen planus [28]. However, regarding the condition of dentine hypersensitivity (DHS) and OHRQoL (and especially the OHIP), the literature is limited.

DHS and its influence on oral health-related quality of life

DHS is an oral complaint frequently reported in clinical dental practice. It is characterised by a short and sharp pain 
that occurs in the presence of thermal, chemical, evaporative, tactile or osmotic stimuli, ceases after their removal and cannot be explained as arising from any other form of dental defect or pathology [29, 30]. From the relatively few studies that have been concerned with the prevalence of DHS, it can be concluded that it is a frequent condition [31]. Depending on the patient group and the study design, prevalences of 4 $57 \%$ up to $60-98 \%$ have been reported [32, 33]. In a national survey in Germany, $39 \%$ of middle-aged adults reported problems with hypersensitive teeth [34]. Pain is the major symptom of the condition. The degree of discomfort depends on the individual's pain perception and pain tolerance, as well as emotional and physical factors. Whereas many affected individuals do not seek treatment to desensitise their teeth because they do not perceive DHS to be a severe oral health problem [35], studies have indicated that a substantial segment of patients (10-25\%) do seek treatment, complaining of different causes of discomfort, such as pain while consuming hot or cold foods and beverages (coffee, ice cream), during toothbrushing or sometimes even while breathing [36, 37]. From the perspective of the patients, these symptoms and problems are highly relevant. The restrictions on everyday activities can have an important effect on their overall quality of life [10]. However, relatively little research on DHS has been reported.

\section{Material and methods}

A review of the literature revealed that few studies about the influence of DHS on OHRQoL have been conducted in any country. A PubMed search and a manual search through the end of October 2011 were conducted using the terms ("dentin sensitivity" [MeSH Terms] OR ("dentin" [All Fields] AND "sensitivity" [All Fields]) OR "dentin sensitivity" [All Fields]) AND (("oral health" [MeSH Terms] OR ("oral" [All Fields] AND "health" [All Fields]) OR “oral health" [All Fields]) AND related [All Fields] AND ("quality of life" [MeSH Terms] OR ("quality" [All Fields] AND "life" [All Fields]) OR "quality of life" [All Fields])) with no language restrictions. Any relevant work published presenting pertinent information about the described issue was considered for inclusion in the review.

\section{Results}

The combination of the search terms resulted in a list of only three titles [38-40]. Two of these articles were published in English, and one was published in German (Table 1). The German publication and one of the English publications were carried out by the same study group. Although the number of articles was limited, a considerable heterogeneity was found between both study groups with regard to study design and patient characteristics. Furthermore, different instruments were used for the assessment of OHRQoL. One study group used the widely used OHIP to describe OHRQoL in patients with DHS and then to measure the efficacy of treatment intervention on OHRQoL in these patients. The other study group developed a completely new instrument, the Dentine Hypersensitivity Experience Questionnaire (DHEQ).

Using the German version of the OHIP, the OHIP-G, Bekes et al. [38] evaluated OHRQoL impairment in patients seeking care for their hypersensitive teeth in comparison with general population subjects $[12,17]$. Study participants were 656 patients without removable prosthodontics who sought treatment for their hypersensitive teeth in German dental offices. These patients were asked to complete the OHIP-G prior to treatment. The sum of OHIP-G item responses (OHIP-G49, 0-196) characterised the OHRQoL impairment. Patients' OHIP summary scores were analysed and compared with those in a sample of the German general population $(n=1,541)$. It was found that the distribution of OHIP-G summary scores in patients with DHS and in general population was different. The general population subjects had an OHIP-G median score of 5, while the patient group had an OHIP-G median score of 30 . The $10 \%$ of the subjects with the highest OHIP-G summary scores presented scores of 36 or higher (general population) and 66 or higher (patients). The mean OHIP-G summary score value of the study participants from the general population was $12.2( \pm 18.4)$, while the patients' mean OHIP-G summary score was $34.5( \pm 22.6)$. The difference was statistically significant $(P<0.001)$. Mean OHIP summary scores indicated that patients with hypersensitive teeth reported considerably more impaired OHRQoL (approximately 22 OHIP units) than subjects in the general population. It was also found that the influence of gender depended on the population (i.e. female general population subjects had lower OHIP scores than male general population subjects, and female patients had higher OHIP scores than male patients). A relationship between age and OHIP-G summary scores was also observed in both patients with hypersensitive teeth and general population subjects. In the general population, older subjects $(40+$ years $)$ reported statistically significantly $(P<0.001)$ more problems (i.e. higher OHIP-G summary scores) than younger subjects (15-39 years). In the patient group, the difference between younger and older patients was similar in magnitude to that in the general population and close to statistical significance $(P=0.08)$.

The results show that DHS is related to substantially impaired OHRQoL. Moreover, this study is the first one evaluating the impact of this oral condition with a widely used patient-centred outcome measure, such as the OHIP, to characterise the broader influence of this condition on patients' perceived oral health. The authors discuss that the 


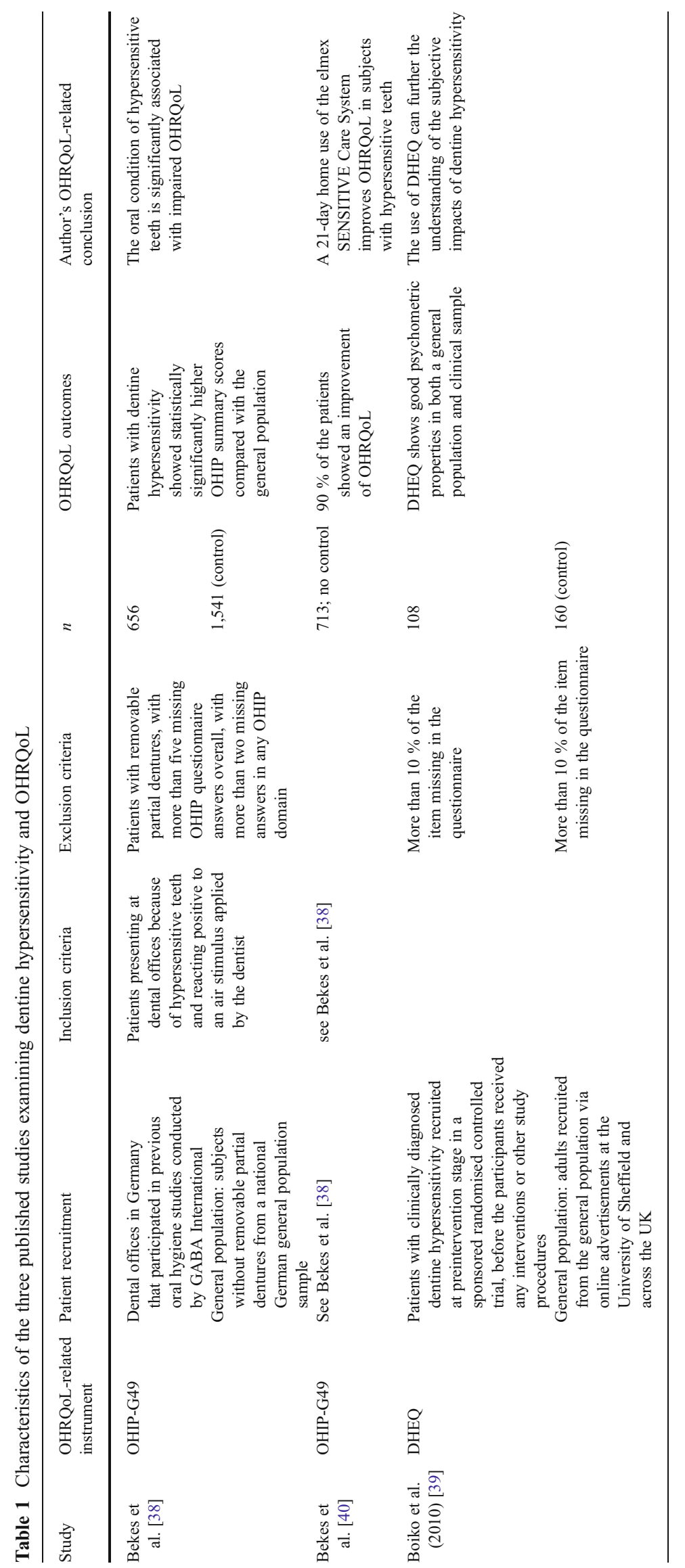


results refer to patients seeking care for their condition and that they cannot be generalised to individuals suffering from DHS but not seeking care. Moreover, they note that it has to be taken into account that the results refer to general dentists and may not be generalised to health professionals who are more trained or calibrated in the assessment of DHS. However, the authors suggest that the setting is representative for typical patients where the general dentist is confronted with providing diagnosis and treatment for the challenging clinical problem of DHS.

In a second investigation, the same study group used the OHIP-G to describe OHRQoL in patients with hypersensitive teeth before and after treatment intervention [40]. In a field trial, 713 patients from Germany presenting at 161 dental offices and seeking care for DHS were given the elmex SENSITIVE Care System to use for their oral hygiene twice a day for 21 days. The system included elmex SENSITIVE toothpaste, elmex SENSITIVE dental rinse and the elmex interX SENSITIVE toothbrush. The patients completed the OHIP-G before and after treatment intervention. The authors found a considerable improvement of OHRQoL in $90 \%$ of patients, with $50 \%$ of the patients showing a decrease of 11 or more OHIP units. The mean change was 13.5 OHIP-G units and was statistically significant $(P<0.001)$. Little difference based on gender or age was present. The results showed that a 21-day home use of the elmex SENSITIVE Care System improved OHRQoL in subjects with hypersensitive teeth. Certainly, the study has limitations. It was not randomised and did not include a control or placebo group. Nevertheless, it is the first study evaluating the effect of a DHS treatment not only from the clinical view of the dentist, but also from the patient's perspective. The results suggest that it is possible to measure the success of a treatment for DHS with the OHIP.

The third study focusing on OHRQoL and DHS found in the literature was conducted by Boiko et al. [39] in the UK. These authors did not use the well-established OHIP as an OHRQoL instrument, but instead developed and validated a new condition-specific measure of OHRQoL for DHS, the DHEQ. This instrument aims to measure particular everyday impacts on OHRQoL related to DHS. The study group constructed a questionnaire using a multistaged impact approach and an explicit theoretical model. As a result, the DHEQ questionnaire has 48 items containing a description of the pain, a scale to capture subjective impacts of DHS, a global oral health rating and a scale to record effects on overall life. The description of the pain contains nine items and each item is treated separately. The impact scale has six subscales based on the initial domains of the Wilson and Cleary model [41]: restrictions, approach coping, avoidance coping, social impact, emotional impact and identity. Two summary measures can be created for the impact scale and its subscales. The total score is calculated as the sum of the item scores per participant (possible range 0-243). Subscale scores for each of the subscales can be created in the same way. The extent of impacts are calculated as the number of impacts per participant to which each participant broadly agrees ("strongly agree" to "strongly disagree"). The global oral health rating contains one item, the scale to record effects on overall life four items. The higher the total score of the DHEQ is, the more impaired is the patient's OHRQoL. In the study, the DHEQ was applied in a general population sample $(n=160)$ and in a sample of patients clinically diagnosed with DHS $(n=108)$. The DHEQ showed good psychometric properties in both samples and demonstrated that patients with DHS had higher scores on the DHEQ than the general population, indicating that these patients have impaired OHRQoL. The authors concluded that the DHEQ provides an alternative to generic OHRQoL instruments because of its direct reference to the problems associated with sensitive teeth, and its use can further the understanding of the subjective impacts of DHS.

As this publication introduced this new instrument and focused on the psychometric properties of the instrument, it has not been applied in any other study yet. In addition, the effects of DHS treatment interventions on OHRQoL have yet to be measured using the DHEQ. Future studies will need to examine this measurement tool translated in other languages so that results can be compared cross-culturally before it will be accepted internationally. Because this instrument is a special measurement tool that can only be used for the condition of DHS, its utility is limited to this special patient group. Therefore, it is not possible - in contrast to the OHIP - to use the DHEQ to measure OHRQoL in patients with other oral diseases.

\section{Conclusion}

The concept of OHRQoL brings a new perspective to clinical care and research. It shifts the focus of clinicians and researchers from the oral cavity alone to the patient as a whole. OHRQoL allows insight into how a patient's oral health affects his/her well-being and quality of life at a given point in time. It can be measured with standardised validated questionnaires (e.g. the OHIP). Thus, different oral diseases and oral complaints can be compared directly.

Although knowledge about the influence of DHS on OHRQoL is incomplete and has not been systematically studied, the following conclusions can be drawn:

- OHRQoL assessment provides additional information about the magnitude of the condition of hypersensitive teeth and should be used in addition to clinical tests to assess the severity and impact of the problem.

- Patients with sensitive teeth report substantial OHRQoL impairment. This finding may have an influence on whether or how patients should be treated. 
- The influence of oral pain, regardless of the location, on subjects' perceived oral health is considerable.

\section{Clinical relevance}

Oral diseases can lead to physical, psychological and social disability. This paper shows that DHS can have a negative impact on the patients' OHRQoL.

Conflict of interest The authors declare that they have no conflict of interest.

\section{References}

1. Slade GD (2002) Assessment of oral health-related quality of life. In: Inglehart MR, Bagramian RA (eds) Oral health-related quality of life. Quintessence, Carol Stream, pp 29-45

2. World Health Organization (1993) Study protocol for the World Health Organization project to develop a Quality of Life assessment instrument (WHOQOL). Qual Life Res 2:153-159

3. Allen PF (2003) Assessment of oral health related quality of life. Health Qual Life Outcomes 1:40

4. Inglehart M, Bagramian RA (2002) Oral health related quality of life: an introduction. In: Inglehart M, Bagramian RA (eds) Oral health related quality of life. Quintessence, Carol Stream, pp 13-28

5. Al Shamrany M (2006) Oral health-related quality of life: a broader perspective. East Mediterr Health J 12:894-901

6. U.S. Department of Health and Human Services (2000) Oral health in America: a report of the Surgeon General U.S. Department of Health and Human Services, National Institute of Dental and Craniofacial Research. National Institutes of Health, Rockville

7. Loe $H$ (1967) The gingival index, the plaque index and the retention index systems. J Periodontol 38(Suppl):610-616

8. Russell AL (1967) The periodontal index. J Periodontol 38 (Suppl):585-591

9. Gruebbel AO (1944) A measurement of dental caries prevalence and treatment service for deciduos teeth. J Dent Res 23:163-168

10. Locker D (1988) Measuring oral health: a conceptual framework. Commun Dent Health 5:3-18

11. Heydecke G, Tedesco LA, Kowalski C, Inglehart MR (2004) Complete dentures and oral health-related quality of life-do coping styles matter? Commun Dent Oral Epidemiol 32:297-306

12. Slade GD, Spencer AJ (1994) Development and evaluation of the oral health impact profile. Commun Dent Health 11:3-11

13. Skaret E, Astrom AN, Haugejorden O (2004) Oral Health Related Quality of Life (OHRQoL) review of existing instruments and suggestions for use in oral health outcome research in Europe. In: Bourgeois DM, Llodra JC (eds) European Global Health Indicators Development Project. Quintessence, Paris, pp 99-110

14. Lopez R, Baelum V (2006) Spanish version of the oral health impact profile (OHIP-Sp). BMC Oral Health 6:11

15. Larsson P, List T, Lundstrom I, Marcusson A, Ohrbach R (2004) Reliability and validity of a Swedish version of the Oral Health Impact Profile (OHIP-S). Acta Odontol Scand 62:147-152

16. Wong MC, Lo EC, McMillan AS (2002) Validation of a Chinese version of the Oral Health Impact Profile (OHIP). Commun Dent Oral Epidemiol 30:423-430
17. John MT, Patrick DL, Slade GD (2002) The German version of the Oral Health Impact Profile - translation and psychometric properties. Eur J Oral Sci 110:425-433

18. Szentpetery A, Szabo G, Marada G, Szanto I, John MT (2006) The Hungarian version of the oral health impact profile. Eur J Oral Sci 114:197-203

19. Schierz O, John MT, Reissmann DR, Mehrstedt M, Szentpetery A (2008) Comparison of perceived oral health in patients with temporomandibular disorders and dental anxiety using oral healthrelated quality of life profiles. Qual Life Res 17:857-866

20. Barros Vde M, Seraidarian PI, Cortes MI, de Paula LV (2009) The impact of orofacial pain on the quality of life of patients with temporomandibular disorder. J Orofac Pain 23:28-37

21. Busato IM, Ignacio SA, Brancher JA, Gregio AM, Machado MA, Azevedo-Alanis LR (2009) Impact of xerostomia on the quality of life of adolescents with type 1 diabetes mellitus. Oral Surg Oral Med Oral Pathol Oral Radiol Endod 108:376-382

22. Souza FT, Santos TP, Bernardes VF, Teixeira AL, Kummer AM, Silva TA, Abreu MH (2011) The impact of burning mouth syndrome on health-related quality of life. Health Qual Life Outcomes 9:57

23. Dueled E, Gotfredsen K, Trab Damsgaard M, Hede B (2009) Professional and patient-based evaluation of oral rehabilitation in patients with tooth agenesis. Clin Oral Implants Res 20:729-736

24. Mulligan R, Seirawan H, Alves ME, Navazesh M, Phelan JA, Greenspan D, Greenspan JS, Mack WJ (2008) Oral health-related quality of life among HIV-infected and at-risk women. Community Dent Oral Epidemiol 36:549-557

25. Tomar SL, Pereyra M, Metsch LR (2011) Oral health-related quality of life among low-income adults living with HIV. J Public Health Dent 71:241-247

26. Allen PF, Thomason JM, Jepson NJ, Nohl F, Smith DG, Ellis J (2006) A randomized controlled trial of implant-retained mandibular overdentures. J Dent Res 85:547-551

27. Heydecke G, Locker D, Awad MA, Lund JP, Feine JS (2003) Oral and general health-related quality of life with conventional and implant dentures. Community Dent Oral Epidemiol 31:161-168

28. Hegarty AM, Hodgson TA, Lewsey JD, Porter SR (2002) Fluticasone propionate spray and betamethasone sodium phosphate mouthrinse: a randomized crossover study for the treatment of symptomatic oral lichen planus. J Am Acad Dermatol 47:271279

29. Addy M (1990) Etiology and clinical implications of dentine hypersensitivity. Dent Clin North Am 34:503-514

30. Canadian Advisory Board on Dentin Hypersensitivity (2003) Consensus-based recommendations for the diagnosis and management of dentin hypersensitivity. J Can Dent Assoc 69:221226

31. Lier BB, Rosing CK, Aass AM, Gjermo P (2002) Treatment of dentin hypersensitivity by Nd:YAG laser. J Clin Periodontol 29:501-506

32. Orchardson R, Collins WJ (1987) Clinical features of hypersensitive teeth. Br Dent J 162:253-256

33. Chabanski MB, Gillam DG, Bulman JS, Newman HN (1997) Clinical evaluation of cervical dentine sensitivity in a population of patients referred to a specialist periodontology department: a pilot study. J Oral Rehabil 24:666-672

34. John MT, LeResche L, Koepsell TD, Hujoel P, Miglioretti DL, Micheelis W (2003) Oral health-related quality of life in Germany. Eur J Oral Sci 111:483-491

35. Gillam DG, Seo HS, Bulman JS, Newman HN (1999) Perceptions of dentine hypersensitivity in a general practice population. J Oral Rehabil 26:710-714

36. Schuurs AH, Wesselink PR, Eijkman MA, Duivenvoorden HJ (1995) Dentists' views on cervical hypersensitivity and their 
knowledge of its treatment. Endod Dent Traumatol 11:240244

37. Gillam DG, Bulman JS, Eijkman MA, Newman HN (2002) Dentists' perceptions of dentine hypersensitivity and knowledge of its treatment. J Oral Rehabil 29:219-225

38. Bekes K, John MT, Schaller HG, Hirsch C (2009) Oral healthrelated quality of life in patients seeking care for dentin hypersensitivity. J Oral Rehabil 36:45-51

39. Boiko OV, Baker SR, Gibson BJ, Locker D, Sufi F, Barlow AP, Robinson PG (2010) Construction and validation of the quality of life measure for dentine hypersensitivity (DHEQ). J Clin Periodontol 37:973-980

40. Bekes K, Schaller HG, Hirsch C (2008) [Verbesserung mundgesundheitsbezogener Lebensqualität durch die Anwendung von Mundhygieneprodukten für schmerzempfindliche und freiliegende Zahnhälse] Improvement of oral health-related quality of Life in subjects with dentin hypersensitivity. ZWR 117:136-142

41. Wilson IB, Cleary PD (1995) Linking clinical variables with health-related quality of life. A conceptual model of patient outcomes. JAMA 273:59-65 